\title{
Low-Frequency Ultrasound for Biofilm Disruption in Chronic Rhinosinusitis With Nasal Polyposis: In Vitro Pilot Study
}

\author{
Tamás Karosi, MD, PhD; István Sziklai, MD, DSc; Péter Csomor, MSc
}

Objectives/Hypothesis: Microbial biofilms have been implicated in the pathogenesis of chronic rhinosinusitis with nasal polyposis (CRSwNP). Although biofilms are characterized by an extremely high resistance against chemical and physical agents, low-frequency ultrasound (LFU) treatment has been suspected to be an efficient and safe method for biofilm disruption.

Study Design: Basic science experimental study.

Methods: A total of 10 patients with CRSwNP undergoing endoscopic sinus surgery were analyzed. Two series of identical nasal polyps $(n=20)$ were processed to hematoxylin-eosin (HE) and Gram staining and to continuous-wave LFU treatment ( 5 minutes, $0.4 \mathrm{MHz}, 37^{\circ} \mathrm{C}$ ), respectively.

Results: Presence of microbial biofilms was confirmed in all patients with CRSwNP. HE staining showed a strong correlation with the results of Gram protocol in biofilm detection. In the LFU-treated group ( $\mathrm{n}=10$ ), a significantly decreased inflammatory cell count was found in the subepithelial layer of nasal polyps $(P<.001)$. In addition, bacterial biofilms were completely removed from the surface of the epithelial layer. Microscopic tissue injuries or significant temperature changes were not detected due to LFU treatment.

Conclusions: Between in vitro conditions, LFU treatment appeared to be a reliable and microscopically safe method for the disruption of microbial biofilms in CRSwNP. These results may provide a basis for a prospective human study investigating the efficacy and safety of this therapeutic modality alone or in combination with antibiotics or topical steroids in biofilmpositive cases of CRSwNP.

Key Words: Biofilm, chronic rhinosinusitis, low-frequency ultrasound, nasal polyps.

Laryngoscope, 000:000-000, 2012

\section{INTRODUCTION}

Chronic rhinosinusitis (CRS) is a common inflammatory disease that is characterized by nasal obstruction, olfactory dysfunction, nasal discharge, and tension pain that persist over 3 months. ${ }^{1,2}$ CRS with nasal polyposis (CRSwNP) is a distinct diagnostic and therapeutic entity with unclarified etiopathogenesis. ${ }^{1,2}$ CRSwNP is currently considered as an immunological disease that is affected by immunological disorders, environmental factors, Staphylococcus superantigens, fungal infections, and the presence of microbial biofilms. ${ }^{1-3}$

Biofilm creates a special environment for microbial survival and proliferation that consists of a self-produced, three-dimensional extracellular matrix formed by polysaccharides, proteins, nucleic acids, and water. $^{4-6}$ Microbial biofilms are characterized by extremely high resistance against antibiotics, host immune reactions,

From the Department of Otolaryngology and Head and Neck Surgery, University of Debrecen, Medical and Health Science Center, Debrecen, Hungary. 9, 2012 .

Editor's Note: This Manuscript was accepted for publication July

The authors have no funding, financial relationships, or conflicts of interest to disclose.

Send correspondence to Tamás Karosi, MD, University of Debrecen, Medical and Health Science Center, Department of Otolaryngology and Head and Neck Surgery, Debrecen, Nagyerdei Krt. 98, H-4032, Hungary. E-mail: karositamas@gmail.com

DOI: 10.1002/lary.23633 and chemical and physical agents. ${ }^{3-5}$ This strong and continuously remodeled physical barrier blocks the diffusion of antibiotics, superoxides, immunoglobulins, and opsonins. ${ }^{3,5,6}$ Biofilms and consecutive inflammatory reactions might contribute to the epithelial damage and subsequent hyperplasia of the subepithelial layer, resulting in nasal polyp formation. ${ }^{6-9}$ Persisting biofilms in CRSwNP may be responsible for surgical failures and high recurrence rate of disease. ${ }^{1,6,7}$ Because biofilms are supposed to play an important role in the pathogenesis of CRSwNP, several therapeutic protocols targeting these structures have been reported and tested in recent years. $^{1,2,10}$

Long-term and low-dose macrolide antibiotic therapy has been supposed to have therapeutic advantages in the elimination of bacterial biofilms in CRSwNP. ${ }^{11,12}$ Beyond the bacteriostatic effects, macrolides have a potential role in the modulation of immune response and consecutive tissue reactions. ${ }^{12-14}$ However, there are conflicting results. In the MACS study (Macrolides in Chronic Rhinosinusitis), low-dose and long-term application of azithromycin did not have any advantages against placebo between clinical conditions. ${ }^{15}$ Because microbial biofilms are characterized by strong negative electrical charge, cationic detergents may have beneficial effects in biofilm disruption. ${ }^{16}$ It has been reported that $1 \%$ baby shampoo has no effects on preformed biofilms; however, it may prevent biofilm formation after endoscopic sinus surgery (ESS) of patients with CRSwNP. ${ }^{16}$ 
In the light of these results, more research is required to investigate the effectiveness of macrolide and cationic detergent therapy against biofilms in CRSwNP.

There is increasing evidence that continuous- (CUS) or pulsed-wave (PUS) low-frequency ultrasound (LFU) treatment is a reliable and safe method for biofilm removal in CRSwNP. ${ }^{10,17-19}$ Bartley and Young discussed that intranasal application of therapeutic ultrasound may increase the sensitivity against antibiotics due to the physical fragmentation of compact biofilm barriers. ${ }^{17}$ Preoperative application of LFU treatment may increase the surgical success rates with decreased frequency of repeated surgeries due to recurring disease. ${ }^{17}$ Young et al. treated 22 CRSwNP patients by $1 \mathrm{MHz}$ pulsed-wave ultrasound 2 to 3 days a week for six sessions. ${ }^{18}$ In contrast to the extranasal application of LFU treatment, the authors have reported a significant improvement of sinusitis symptoms in 18 patients according to the decreased values of Sino-Nasal Outcome Test questionnaire. ${ }^{18}$ Later, Ansari et al. reported a significant difference between the effectiveness of pulsedwave (PUS) and continuous-wave (CUS) ultrasound therapy in CRS. ${ }^{20}$ The authors found a greater improvement of sinusitis-associated symptoms in the PUS-treated patient group. ${ }^{20}$ In a preliminary study on cadaver heads, Patel et al. demonstrated that LFU treatment is a safe protocol that was confirmed by histopathological analysis of the ultrasound-treated nasal mucosa. ${ }^{19}$ This research group also demonstrated that LFU treatment may promote the delivery of various topical solutions in the nasal cavity, except for the frontal sinus. ${ }^{19}$

This preliminary study investigates the effectiveness and microscopic safety of low-frequency continuouswave ultrasound treatment in biofilm disruption in chronic rhinosinusitis with nasal polyposis between in vitro conditions.

\section{MATERIALS AND METHODS}

\section{Patients}

A case-control experimental study was performed on nasal polyp specimens obtained from patients with CRSwNP who underwent ESS at the Department of Otorhinolaryngology and Head and Neck Surgery, University of Debrecen. Nasal polyps $(\mathrm{n}=20)$ were collected between January 2012 and April 2012. The patient group consisted of four males and six females $(n=10$, mean age, 51.3 years; range, 32-61 years) with the diagnosis of CRSwNP confirmed by nasal endoscopy and computed tomography (CT) scans of the paranasal sinuses. All the nasal polyps collected during ESS were divided into two consecutive groups consisting of 1010 specimens that were processed to histopathological analysis alone and LFU treatment, respectively. Only nasal polyps larger than $2 \mathrm{~cm}$ were analyzed, which could be removed by straight endoscopic forceps without any surface injury or iatrogenic disruption of biofilm layers. The removal was gently performed at the root of the nasal polyps. All patients gave their informed consent before donating their tissue samples for the study. The institutional ethical committee approved our study. The study was carried out according to the Declaration of Helsinki.

\section{Hematoxylin-Eosin and Gram Staining}

According to previous reports, a combination of hematoxylin-eosin (HE) and Gram staining protocols seems to be a usable method for the detection of biofilm existence and corresponding histopathological changes, because HE staining is for the investigation of microscopic architecture, whereas Gram protocol stains various microbial elements. ${ }^{21,22}$ The first series of 10 LFU-untreated nasal polyps were fixed in $10 \%$ (w/v) formaldehyde. Specimens were embedded in $15 \%(\mathrm{w} / \mathrm{v})$ purified gelatin $\left(24\right.$ hours, $\left.56^{\circ} \mathrm{C}\right)$ and refixed in $4 \%(\mathrm{w} / \mathrm{v})$ paraformaldehyde $(24$ hours, $20^{\circ} \mathrm{C}$ ). Blocks were cryoprotected in $20 \%$ (w/v) sucrose solution $\left(2\right.$ hours, $4^{\circ} \mathrm{C}$ ) and sectioned into 5 - $\mu \mathrm{m}$ slides at $-25^{\circ} \mathrm{C}$ (MNT-200; Slee, Mainz, Germany). Slides were stored in $0.1 \mathrm{M}$ phosphate-buffered saline containing $0.03 \%$ (w/v) sodium azide at $4^{\circ} \mathrm{C}$. Two consecutive $5-\mu \mathrm{m}$ frozen-cut sections were examined as follows: 1) conventional staining with $\mathrm{HE}$, and 2) conventional Gram staining. The criteria for the histopathological detection of microbial biofilms were the presence of characteristic morphology and Gram positivity/negativity and microcolonies for examination by optical microscopy and the presence of the surrounding polysaccharide layer. ${ }^{22}$ Structure and cellular infiltration of the epithelial and also the subepithelial layers were correlated to the presence of bacterial biofilms.

\section{In Vitro LFU Treatment}

The second series of nasal polyps were placed into $0.9 \%$ $(\mathrm{w} / \mathrm{v})$ sodium chloride solution immediately after surgical removal. LFU treatment protocol was started within 30 minutes after tissue sampling (Sonica 5200-EP; Soltec, Milan, Italy). Nasal polyp specimens were placed into a self-designed silicone vat. $\mathrm{LFU}$ treatment with $0.4 \mathrm{MHz}$ frequency and $0.5 \mathrm{~W} / \mathrm{cm}^{2}$ sound pressure was employed for 5 minutes at $37^{\circ} \mathrm{C}$ in $20 \mathrm{~mL}$ total volume of $0.9 \%(\mathrm{w} / \mathrm{v})$ sodium chloride solution. A digital thermometer was applied to register any temperature changes during the protocol. Ultrasound-treated nasal polyps were fixed in $10 \%(\mathrm{w} / \mathrm{v}$ ) formaldehyde and then the same histopathological staining protocols were performed that were previously described. Histopathological characteristics of biofilms, and epithelial and subepithelial layers of untreated specimens were correlated to ultrasound-treated identical nasal polyp pairs with the aim of analyzing the effects of therapeutic ultrasound on biofilms and microscopic structures. Statistical assessments were performed by Mann-Whitney $U$ probe with a $95 \%$ confidence interval (SPSS 9.0 for Windows; SPSS, Inc. Chicago, IL). Histological pretreatment protocols were performed by an independent laboratory assistant in all cases. Both sets of histological examinations in the LFU-treated and untreated sample groups were independently analyzed by two researchers (P.C. and T.K.)

\section{RESULTS}

Altogether, 10 patients with CRSwNP who underwent ESS were included in this study. The LundMackay scores of coronal reconstructed CT scans varied between 15 and 24 with an average score of 21 , indicating serious involvement of paranasal sinuses by CRS. The score was higher than 21 in $70 \%$ of patients $(n=7)$. The clinical history and findings of physical examinations were obtained during confirmation of the diagnosis of CRSwNP. Clinical information on bronchial asthma, allergic rhinitis, aspirin intolerance, previous ESS, topical steroid treatment, and systemic antimicrobial therapy was recorded before surgery. Three patients $(30 \%)$ had bronchial asthma, 10\% $(\mathrm{n}=1)$ had allergic rhinitis, and $10 \%(\mathrm{n}=1)$ had the diagnosis of aspirin intolerance. This patient had complete acetylsalicylic 


\section{No ultrasound treatment}
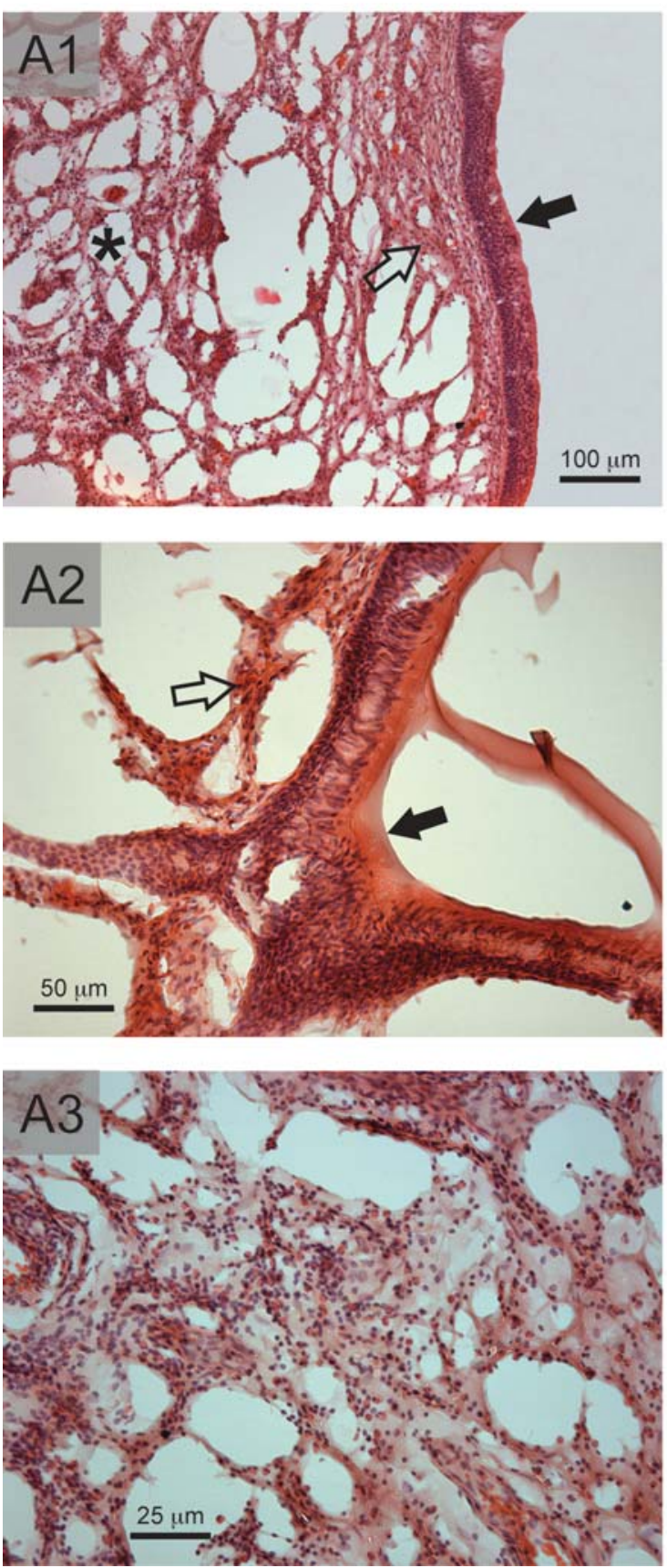

Ultrasound treatment $(5 \mathrm{~min}, 40 \mathrm{kHz})$
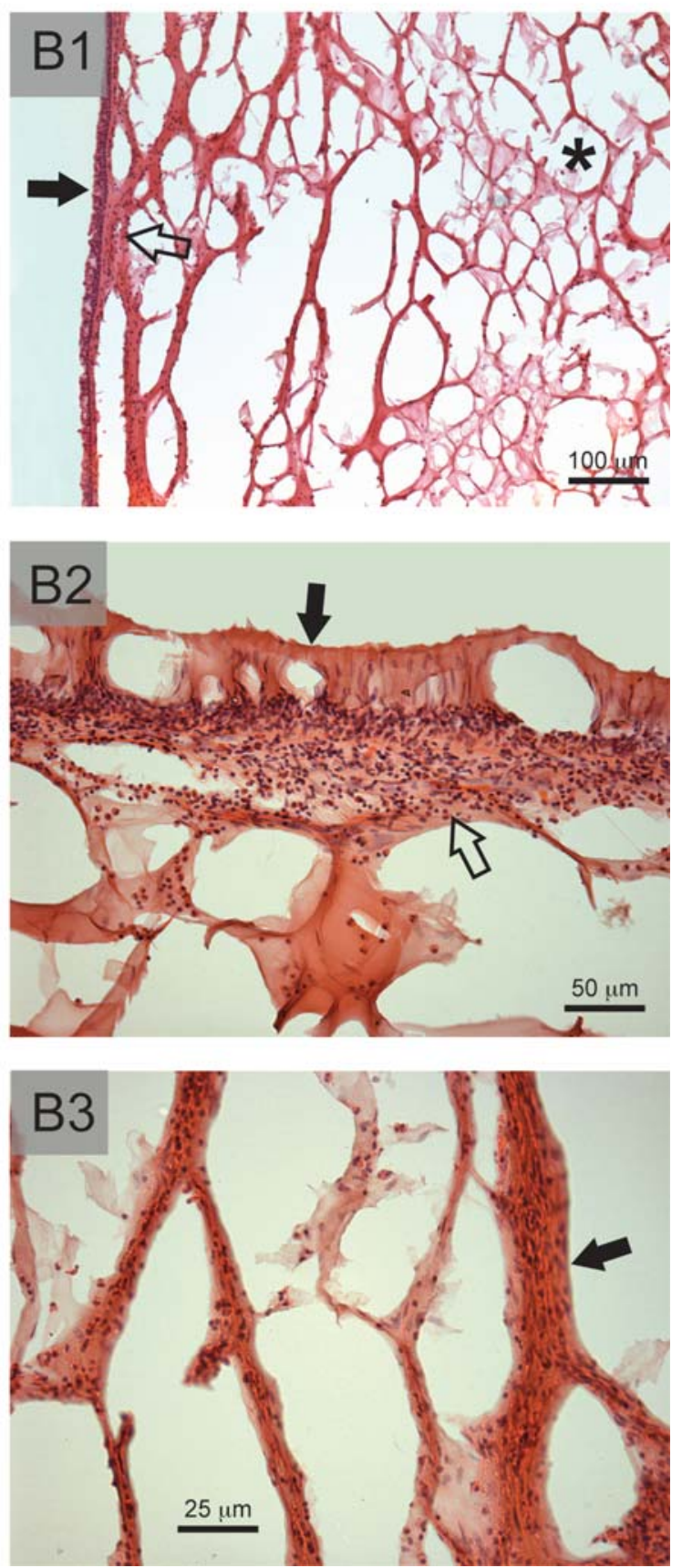

Fig. 1. Microphotographs of nasal polyps stained by hematoxylin-eosin before and after low-frequency ultrasound treatment. (A1) At low magnification, a columnar epithelium with eosinophilic polysaccharide layer can be detected (black arrow). The submucosal layer is marked by a white arrow, whereas the black asterisk indicates the stromal layer with massive infiltration of inflammatory cells. (A2) Microbial biofilm is displayed as a dense, eosinophilic structure on the surface of epithelial layer (black arrow). The white arrow shows the submucosa. (A3) Stromal substance of the nasal polyp is heavily infiltrated by eosinophil and neutrophil granulocytes and also by plasmocytes. (B1) At low magnification, a columnar epithelium without biofilm-like structures can be detected (black arrow). The submucosal layer is marked by a white arrow, whereas black asterisk indicates the hypocellular stromal layer. (B2) The respiratory epithelium shows intact architecture without any signs for superficial biofilm presence (black arrow). The white arrow shows the intact submucosal layer. (B3) The stromal layer represents as a hypocellular structure with sporadic inflammatory cells and intact connective tissue web.

acid triad (nasal polyposis, bronchial asthma, and aspirin intolerance). Diagnosis of allergic rhinitis was based on the clinical history, on the physical examination, and on the allergen-specific intracutaneous (prick) skin test. Inhalative allergen-specific serum immunoglobulin
$\mathrm{G}$ levels were also measured by enzyme-linked immunosorbent assay in all cases. The diagnosis of bronchial asthma was based on the clinical history and on the respiratory functional test. The final diagnosis was stated by an experienced pulmonologist in all cases. Aspirin 
No ultrasound treatment
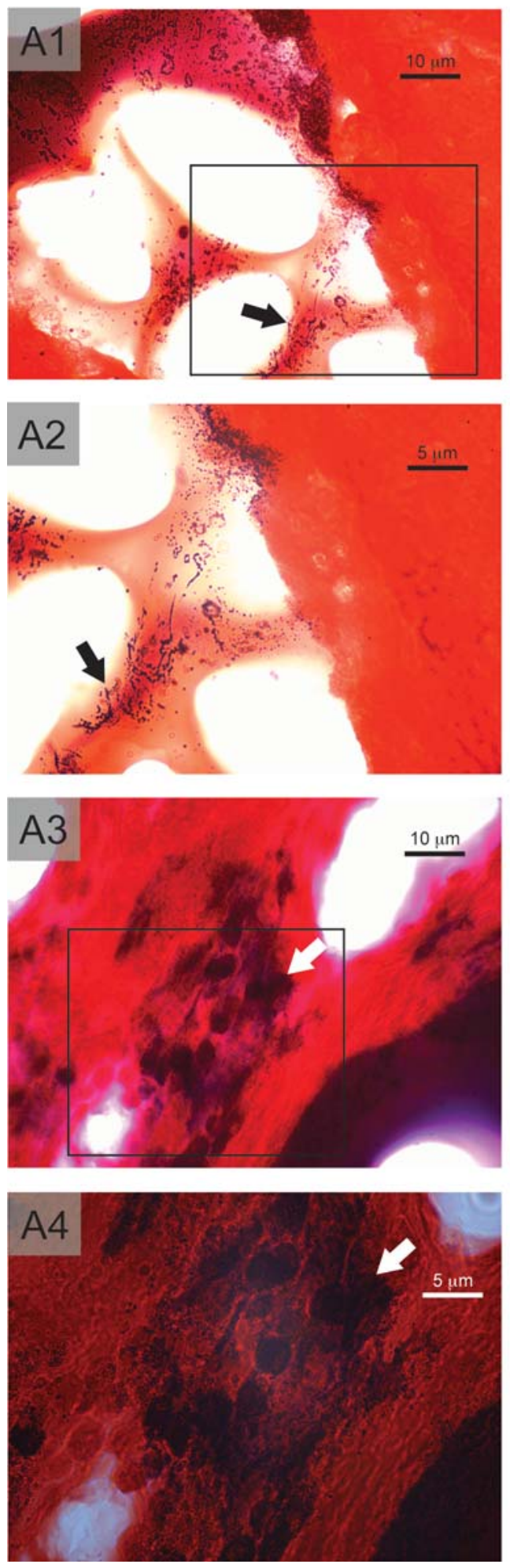

Ultrasound treatment (5 min, $40 \mathrm{kHz}$ )
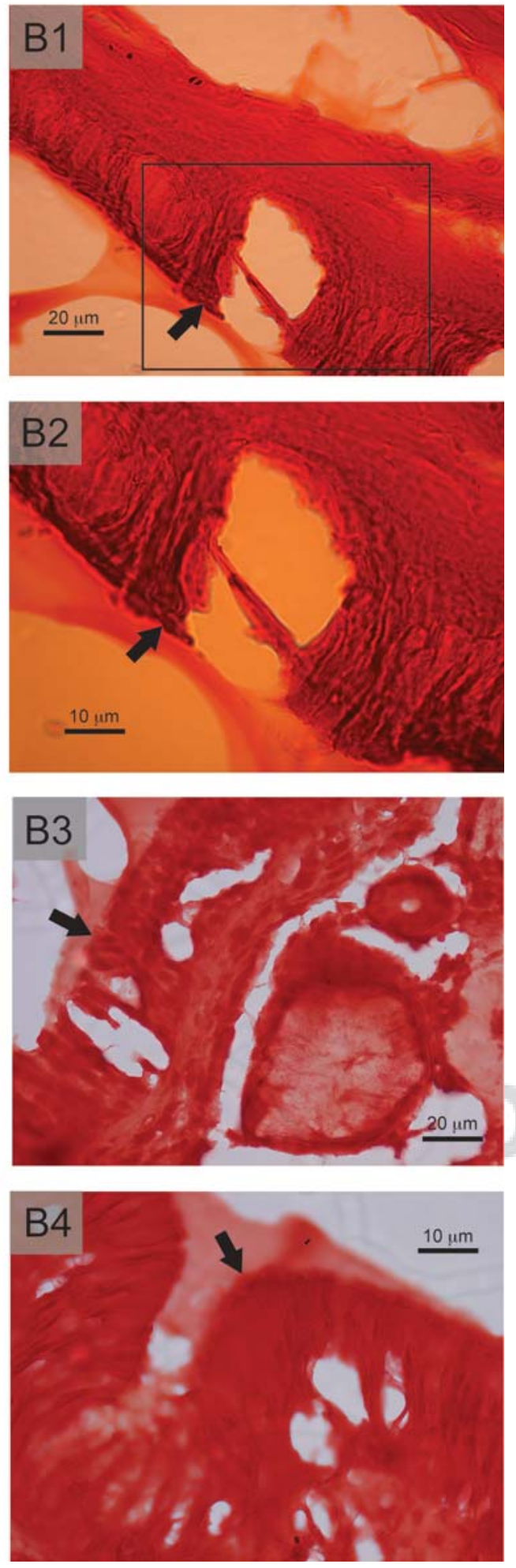

C

Fig. 2. Microphotographs of Gram-stained nasal polyps before and after low-frequency ultrasound treatment. (A1) Gram staining indicates a bacterial biofilm consisting of several chains of Gram-positive cocci (black arrow). (A2) Higher magnification view of the previous section. The black arrow shows bacteria of 0.3 to $0.5 \mu \mathrm{m}$ in size. (A3) Gram staining reveals a bacterial biofilm with intraepithelial invasion of Gram-positive cocci (white arrow). (A4) At higher magnification, a serious intracellular bacterial infiltration can be detected in the epithelial layer (white arrow). (B1) Gram staining is negative for bacterial elements; the polysaccharide matrix is absent (black arrow). (B2) Higher magnification view of the previous section. (B3) The epithelial layer is intact; biofilm cannot be detected (black arrow). (B4) Higher magnification view of the previous specimen. intolerance was based on the presence of aspirininduced hypersensitive reactions in the clinical history of the patients. Repeated ESS was performed in 50\% (n $=5$ ) of patients, which is an important predictive factor of CRS recurrence. In this group, number of previous surgeries varied between two to four, with an average number of three ESSs. All patients were treated by topical mometasone furoate monohydrate (200 $\mu \mathrm{g} /$ day, Nasonex; Merck-Schering-Plough, Whitehouse Station, NJ) therapy before surgery. The average period of intranasal steroid treatment was 22.8 months, which varied between 11 to 63 months. Preoperative 
TABLE I.

Histological Parameters of Biofilm Thickness and Subepithelial Cellular Infiltration in Nasal Polyps According to Low-Frequency Ultrasound Treatment.

\begin{tabular}{|c|c|c|c|}
\hline Nasal Polyp Specimens $(n=20)$ & LFU-Untreated Group $(n=10)^{*}$ & LFU-Treated Group $(n=10)$ & $P$ Value $^{\dagger}$ \\
\hline Biofilm thickness $(\mathrm{mm})^{\ddagger}$ & $17.3(9.24-22.53)$ & $0(0)$ & $<.001$ \\
\hline Inflammatory cell count in the stromal layer ${ }^{\S}$ & $603.72(486-730.4)$ & $38.81(17.5-71.3)$ & $<.001$ \\
\hline
\end{tabular}

*Low-frequency ultrasound (LFU) treatment between in vitro conditions (5 minutes, $37^{\circ} \mathrm{C}, 0.4 \mathrm{MHz}$ ).

${ }^{\dagger} P$ value at $95 \%$ confidence interval (Mann-Whitney $U$ probe).

${ }^{\ddagger}$ Mean value of biofilm thickness in the average of 10 large magnification microscopic fields (range).

$\S$ Mean value of inflammatory cell number (lymphocyte, plasmocyte, neutrophil, basophil, and eosinophil granulocyte) in the average of 10 large magnification microscopic fields (range).

(within 6 weeks), systemic antibiotic treatment (cefuroxime) was performed in one $(10 \%)$ patient due to acute rhinosinusitis.

The histopathological examination revealed inflammatory nasal polyps with polymorphonuclear and plasmocyte infiltration of the subepithelial layer in all

F1 cases (Fig. 1). Disintegrated epithelial layer with partial squamous cell metaplasia was found in seven cases $(\mathrm{n}=$ $7,70 \%)$, whereas in three nasal polyps $(\mathrm{n}=3,30 \%)$ the epithelium showed normal architecture with ciliated columnar and foamy cells (Fig. 1). Bacterial biofilms were

F2 detected in all patients with CRSwNP (Figs. 1 and 2, Ta-

$\mathrm{T} 1$ ble I). In our series, HE staining displayed a strong correlation with the results of Gram staining and was found to be a reliable predictor of the presence or absence of biofilms. In the LFU-untreated group, thickness of biofilm layer varied between 9.24 to $22.53 \mu \mathrm{m}$, with the average of $17.3 \mu \mathrm{m}$ (Table I). In the LFU-treated group of nasal polyps, combined application of $\mathrm{HE}$ and Gram-staining protocols revealed the total absence of bacterial biofilms $(P<.001)$ (Figs. 1 and 2, Table I). In the LFU-untreated nasal polyps, the stromal substance displayed a serious inflammatory cell infiltration that was predominantly formed by eosinophil and neutrophil granulocytes (Fig. 1). In the average of 10 large magnification microscopic fields, the inflammatory cell count varied between 486 to 730.4 , with the average of 603.72 cells (Fig. 1, Table I). In the LFU-treated group, the average number of inflammatory cells decreased to 38.81 , which was a statistically significant difference $(P<.001)$ (Fig. 1, Table I). Architectural changes due to LFU treatment were not detected in the epithelial or in the subepithelial layers of nasal polyps (Fig. 1). In the LFUtreated group, the reticular pattern of the connective tissue web remained intact, and no signs of vascular damage, leukocyte disintegration, or karyorrhexis (leukocytoclasia) could be detected (Fig. 1). No significant temperature changes were detected during LFU treatment (average $\Delta \mathrm{T}$ was $0.34^{\circ} \mathrm{C}$ ).

\section{DISCUSSION}

In the present study, we demonstrated the presence of biofilms in all patients with CRSwNP by the combined application of HE and Gram protocols. ${ }^{22}$ The weakness of our study is the absence of a control group and the relatively low number of subjects, which should be increased in the future to obtain more precise statistical correlations. Although the presence of biofilm itself is supposed to be an important factor in the pathogenesis of CRSwNP, microbiological identification of different bacterial and fungal species involved in biofilm formation still requires culturing, confocal laser scanning microscopy, or fluorescent in situ hybridizations analysis with species-specific oligonucleotide probes. ${ }^{4,23}$

The presence of microbial biofilms is suspected to affect the intensity of subepithelial inflammatory reaction in CRSwNP. ${ }^{6-8}$ Inflammatory cells accumulating in the subepithelial and stromal layer of nasal polyps are responsible for the uncontrolled release of several proinflammatory cytokines. ${ }^{8,9,13}$ Increased expression of IL-1, tumor necrosis factor- $\alpha$, receptor activator of nuclear factor kappa-B ligand, and macrophage colony-stimulating factor may lead to epithelial disintegration and increased connective tissue formation in the stromal substance. $^{7,9,13,14}$ Because biofilms might play a central role in chronic inflammation and nasal polyp formation; disruption of these microbial structures seems to be essential in the nonsurgical treatment of CRSwNP. ${ }^{3,6,10}$

CUS or PUS low-frequency ultrasound treatment has been reported as a potent therapeutic option for the physical disruption of biofilms in CRSwNP. ${ }^{10,17,18}$ The idea is not new and has physical evidence, because LFU is widely used for the cleaning of laboratory devices with superficial biofilm contamination. ${ }^{10}$ Therapeutic ultrasound of 0.4 to $3.3 \mathrm{MHz}$ frequencies is commonly used in the rheumatology practice and in rehabilitation medicine. In soft tissues, maximum energy absorption varies between 2 to $4 \mathrm{~cm}$. Therapeutic ultrasound has various tissue effects: it increases blood flow in the treated area, decreases edematous swelling, and results in microcavitation and acoustic streaming. ${ }^{19,20}$ Microcavitation originates from the ultrasound-induced vibration of soft tissues that results in the formation of microscopic air bubbles, which transmit these vibrations directly to the cell membranes, vascular walls, and to biofilms. ${ }^{17,18}$

According to our results, LFU treatment can be supposed as a reliable and safe method for biofilm disruption in CRSwNP. It was found that the amount of inflammatory cells in the subepithelial and stromal layers showed a statistically significant decrease due to LFU treatment. Ultrasound-induced movement, intravascular microstreaming, and the escape effect of leukocytes at the surgical resection surface of nasal polyps might be responsible for this finding. At this point, it seems to be an interesting observation; however, its 


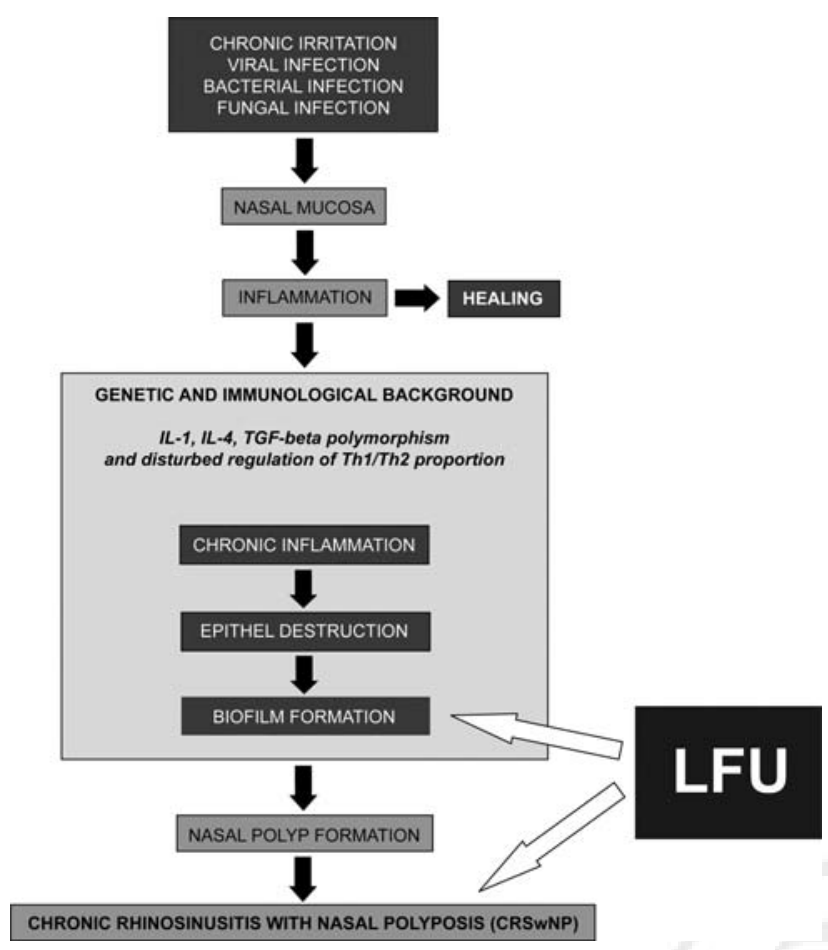

Fig. 3. Schematic model for the potential targets of low-frequency ultrasound (LFU) in chronic rhinosinusitis with nasal polyposis $(\mathrm{CRSwNP}) . \mathrm{IL}=$ interleukin, TGF $=$ transforming growth factor; Th $=\mathrm{T}$ helper.

clinical effects are unpredictable. This unclarified phenomenon may have beneficial effects in the treatment of CRSwNP by a significant reduction of subepithelial inflammation in the nasal mucosa. On the other hand, an uncontrolled release of inflammatory cytokines and consecutive inflammatory reactions with serious rebound effects are also conceivable. Although no leukocyte destruction was found, the answer requires further research between controlled clinical conditions.

According to enhanced wave reflection at tissue borders and limited penetration depth, extranasal application of LFU protocol seems to be only a symptomatic treatment in $\mathrm{CRSwNP}^{18}$ In the near future, intranasal application of LFU treatment will need to be

F3 tested in biofilm disruption in CRSwNP (Fig. 3). To avoid sound reflection that occurs at air interfaces in the nasal cavity, a transducing medium is necessary for the ultrasound energy to be delivered to the mucosal surfaces. We do think that after intranasal upload of $0.9 \%$ $(\mathrm{w} / \mathrm{v})$ sodium chloride solution, application of a thin and special low-frequency ultrasound probe with potential temperature and sound pressure control will be a reliable method in the nonsurgical treatment of CRSwNP (Fig. 3). Certainly, this hypothesis requires several further research and clinical studies in the future.

Corticosteroids have beneficial effects on the symptoms of CRSwNP and significantly decrease nasal obstruction and olfactory dysfunction..$^{1,2,13}$ On the other hand, steroid treatment may have adverse effects on biofilm presence due to the inhibition of local immune response. ${ }^{13,22}$ As we have concluded previously, steroid and systemic antibiotic treatment might adversely affect the structure and thickness of biofilms. ${ }^{22}$ However, a combination of topical steroid medication, systemic antibiotic treatment, and LFU protocol may have additional beneficial effects in the nonsurgical treatment of CRSwNP. In the future, placebo-controlled, doubleblinded, multicenter studies will be necessary to prove the effectiveness of this combination of different therapeutic protocols.

\section{CONCLUSION}

This study indicates a potential therapeutic effect of LFU treatment in CRSwNP, because bacterial biofilms were completely removed in the LFU-treated group of nasal polyps. In general, therapeutic considerations can be divided into two main groups: blockade of biofilm formation and eradication of biofilms that have already formed (Fig. 3). Although specific treatments are not yet available to target microbial biofilm, its detection and eradication is an important requirement, because it is strongly associated with treatment failures and persisting symptoms of CRSwNP. In the future, systemic double-blinded studies will be required to confirm the therapeutic effectiveness of the LFU-protocol in the nonsurgical treatment of CRSwNP.

\section{BIBLIOGRAPHY}

1. Meltzer EO, Hamilos DL. Rhinosinusitis diagnosis and management for the clinician: a synopsis of recent consensus guidelines. Mayo Clin Proc 2011;86:427-443.

2. Marple BF, Stankiewicz JA, Baroody FM, et al.; American Academy of Otolaryngic Allergy Working Group on Chronic Rhinosinusitis. Diagnosis and management of chronic rhinosinusitis in adults. Postgrad Med 2009;121:121-139.

3. Mladina R, Poje G, Vukovic K, Ristic M, Music S. Biofilm in nasal polyps. Rhinology 2008;46:302-307.

4. Ferguson BJ, Stolz DB. Demonstration of biofilm in human bacterial chronic rhinosinusitis. Am J Rhinol 2005;19:452-457.

5. Zernotti ME, Angel Villegas N, Roques Revol M, et al. Evidence of bacterial biofilms in nasal polyposis. J Investig Allergol Clin Immunol 2010; 5:380-385.

6. Al-Mutairi D, Kilty SJ. Bacterial biofilms and the pathophysiology of chronic rhinosinusitis. Curr Opin Allergy Clin Immunol 2011;11:18-23.

7. Galli J, Calo L, Ardito F, Imperiali M, et al. Damage to ciliated epithelium in chronic rhinosinusitis: what is the role of bacterial biofilms? Ann Otol Rhinol Laryngol 2008;117:902-908.

8. Otto BA, Wenzel SE. The role of cytokines in chronic rhinosinusitis with nasal polyps. Curr Opin Otolaryngol Head Neck Surg 2008;16:270-274.

9. Hekiert AM, Kofonow JM, Doghramji L, et al. Biofilms correlate with TH1 inflammation in the sinonasal tissue of patients with chronic rhinosinusitis. Otolaryngol Head Neck Surg 2009;141:448-453.

10. Desrosiers M, Myntti M, James G. Methods for removing bacterial biofilms: in vitro study using clinical chronic rhinosinusitis specimens. Am J Rhinol 2007;21:527-532.

11. Videler WJ, van Hee K, Reinartz SM, Georgalas C, van der Meulen FW, Fokkens WJ. Long-term low-dose antibiotics in recalcitrant chronic rhinosinusitis: a retrospective analysis. Rhinology 2012;50:45-55.

12. Park CS, Park YS, Park YJ, Cho JH, Kang JM, Kim SY. The inhibitory effects of macrolide antibiotics on bone remodeling in chronic rhinosinusitis. Otolaryngol Head Neck Surg 2007;137:274-279.

13. Lennard CM, Mann EA, Sun LL, Chang AS, Bolger WE. Interleukin-1 beta, interleukin-5, interleukin-6, interleukin-8, and tumor necrosis factor-alpha in chronic sinusitis: response to systemic corticosteroids. Am J Rhinol 2000;14:367-373.

14. Kato A, Peters A, Suh L, et al. Evidence of a role for B cell-activating factor of the TNF family in the pathogenesis of chronic rhinosinusitis with nasal polyps. J Allergy Clin Immunol 2008;121:1385-1392.

15. Videler WJ, Badia L, Harvey RJ, et al. Lack of efficacy of long-term, lowdose azithromycin in chronic rhinosinusitis: a randomized controlled trial. Allergy 2011;66:1457-1468.

16. Chiu AG, Palmer JN, Woodworth BA, et al. Baby shampoo nasal irrigations for the symptomatic post-functional endoscopic sinus surgery patient. Am J Rhinol 2008;22:34-37.

17. Bartley J, Young D. Ultrasound as a treatment for chronic rhinosinusitis. Med Hypotheses 2009;73:15-17. 
18. Young D, Morton R, Bartley J. Therapeutic ultrasound as treatment for chronic rhinosinusitis: preliminary observations. J Laryngol Otol 2010;124:495-499.

19. Patel ZM, Hwang PH, Chernomorsky A, et al. Low-frequency pulsed ultrasound in the nasal cavity and paranasal sinuses: a feasibility and distribution study. Int Forum Allergy Rhinol. In press.

20. Ansari NN, Fathali M, Naghdi S, Hasson S, Jalaie S, Rastak MS. A randomized, double-blind clinical trial comparing the effects of continuous and pulsed ultrasound in patients with chronic rhinosinusitis. Physiother Theory Pract 2012;28:85-94.
21. Hochstim CJ, Choi JY, Lowe D, Masood R, Rice DH. Biofilm detection with hematoxylin-eosin staining. Arch Otolaryngol Head Neck Surg 2010;136:453-456.

22. Toth L, Csomor P, Sziklai I, Karosi T. Biofilm detection in chronic rhinosinusitis by combined application of hematoxylin-eosin and gram staining Eur Arch Otorhinolaryngol 2011;268:1455-1462.

23. Psaltis AJ, Ha KR, Beule AG, Tan LW, Wormald PJ. Confocal scanning laser microscopy evidence of biofilms in patients with chronic rhinosinusitis. Laryngoscope 2007;117:1302-1306. 